\title{
The method of calculation and selection of methods of tractor maintenance, taking into account the working conditions of the operator
}

\author{
M.V. Chubareva* and N.V. Chubareva \\ Irkutsk State Agricultural University named after A.A. Ezhevsky, Molodezhniy settlement, Irkutsk region, 664038, Russia
}

\begin{abstract}
The method involves calculation and selection of methods of tractor maintenance, taking into account the working conditions of the operator in the field. It is intended for agricultural enterprises with an average annual number of tractors not exceeding 20 units. Calculation for the choice of maintenance methods is carried out on a personal computer in Microsoft Office Excel or a calculator for the minimum value of the unit cost of tractor maintenance. Research objective is to determine the most rational method of maintenance by the specific cost of tractor maintenance. The method allows determining the unit cost of maintenance, either taking into account the additional costs of tractor maintenance when servicing tractors in the field conditions, or without taking them into account. The article presents the selection procedure for maintenance methods, taking into account the working conditions. The annual economic effect from the application of this method, calculated on the example of agricultural enterprises of the Irkutsk region, is 27.8 thousand rubles per tractor. The method was tested as exemplified by farms in the Tulunsky and Cheremkhovsky districts of the Irkutsk region and can be useful to engineers maintaining farming tractors. The results of the study can be used in agricultural enterprises, both the Irkutsk region and the Siberian region as a whole.
\end{abstract}

\section{Introduction}

Nowadays, many types of research projects are conducted in the field of tractor maintenance - textbooks and teaching aids, dissertations and scientific reports, abstract collections, monographs, articles, theses, etc. This indicates that this area of knowledge is always relevant and in demand [1].

In the article, $\mathrm{R}$ Khodabakhshian [2] gives this brief introduction to various preventive maintenance systems specially condition-based maintenance (CBM) techniques, selection of condition monitoring techniques and understanding of condition monitoring (CM) intervals, advancement in CBM, standardization of CBM system, CBM approach on agricultural machinery, advantages and disadvantages of CBM.

Authors A Rokhani, S Abdolahpur [3] make a forecast on the cost of repair and maintenance based on the artificial neural network (ANN) method, which has a significant impact on making the right economic decisions by controlling mechanisms, such as equipment replacement.

The article by C J A Ter Berg, G Leontaris, M van den Boomen et al. [4] covers the method for the determination of the optimal moment at which maintenance work should be performed with a quality guarantee. The proposed method utilizes Cooke's classical model, which uses knowledge and expert experience, which is estimated according to their performance in judging uncertainty, to assess this period. A bridge-based experiment shows that the proposed method has the potential to provide means to effectively plan maintenance.

Authors Kubeev E.I., Panova S.V. Geyvandov D.B., Shishkin M.A., Khakimov R.T. considered issues related to reducing labor costs for maintenance of vehicles. They argue that reducing labor intensity and increasing reliability has a significant impact on the performance of tractors, as well as on the cost of tractor work. The methods that contribute to reducing the complexity during the maintenance of cars (tractors) are considered, which, ultimately, leads to an increase in their performance [5].

The search for publications on the topic of choosing tractor maintenance methods has not yielded results, therefore, the development of a methodology for choosing tractor maintenance methods has been deemed relevant.

The method is intended for agricultural enterprises with an average annual number of tractors not exceeding 20 units. It provides for the calculation and choice of tractor maintenance methods, taking into account the working conditions of the operator in the field. It is based on the determination of the unit cost of maintenance on the maintenance methods, which include: centralized, decentralized and combined. The

Corresponding author: chubarevamarina@ rambler.ru 
choice of method is carried out according to a minimum of the weighted average unit cost of service by tractor brands.

To calculate the unit cost of tractor maintenance, the following initial data are needed: data on agricultural enterprises, labor and maintenance costs of tractor maintenance, production conditions for the use of tractors and mobile maintenance units for the purpose, as well as the working conditions of the operator. Calculation of the choice of maintenance methods is carried out on a personal computer in Microsoft Office Excel or a calculator.

Research objective is to determine the most rational method of maintenance by the specific cost of tractor maintenance.

\section{Materials and research methods}

Mathematical models for selecting maintenance methods are presented in three stages [6].

The first stage is the mathematical descriptions of maintenance methods in expanded form in the following order:

1. Centralized: maintenance- 1 and maintenance- 2 are carried out at the point of maintenance where vehicles arrive under the own steam

$$
\begin{aligned}
& C_{1}= C_{M P}^{M 1}+C_{M P}^{M 2}+C_{F M P}^{M 1}+C_{F M P}^{M 2}+C_{T M P}^{M 1}+C_{T M P}^{M 2}+C_{I T M P}^{M 1} \\
&+C_{I T M P}^{M 2}+C_{T D M P}^{M 1}+C_{T D M P}^{M 2}= \\
&=\frac{L_{M 1} C_{H W}}{\tau_{M 1}}+\frac{L_{M 2} C_{H W}}{\tau_{M 2}}+\frac{l_{M 1} S_{q M P}}{\tau_{M 1}}+\frac{l_{M 2} S_{q M P}}{\tau_{M 2}}+\frac{2 R_{0} S_{T}}{U_{H E} \tau_{M 1}}+ \\
& \frac{2 R_{0} S_{T}}{U_{H E} \tau_{M 2}}+\frac{l_{M 1} U_{H E}}{\tau_{M 1}}+\frac{l_{M 2} U_{H E}}{\tau_{M 2}}+\frac{2 R_{0} U_{H E}}{V_{T} \tau_{M 1}}+\frac{2 R_{0} U_{H E}}{V_{T} \tau_{M 2}}
\end{aligned}
$$

or after simple transformations

$$
\begin{gathered}
C_{1}=\frac{L_{M 1} C_{H W}+l_{M 1} S_{q M P}+l_{M 1} U_{H E}}{\tau_{M 1}}+\frac{L_{M 2} C_{H W}+l_{M 2} S_{q M P}+l_{M 2} U_{H E}}{\tau_{M 2}}+ \\
+\frac{2 R_{0}}{V_{T}}\left(S_{T}+U_{H E}\right)\left(\frac{1}{\tau_{M 1}}+\frac{1}{\tau_{M 2}}\right),
\end{gathered}
$$

at $L_{M 1}>0, L_{M 2}>0, l_{M 1}>0, l_{M 2}>0, C_{H W}>0, S_{q M P}>0$, $\tau_{M 1}>0, \tau_{M 2}>0, R_{0}>0, V_{T}>0, S_{T}>0, U_{H E}>0$.

2. Decentralized: maintenance-1 and maintenance-2 are performed at the place of tractor operation, using a maintenance unit

$$
\begin{gathered}
C_{2}=C_{M U}^{M 1}+C_{M U}^{M 2}+C_{F M U}^{M 1}+C_{F M U}^{M 2}+C_{T M U}^{M 1}+C_{T M U}^{M 2}+C_{I T M U}^{M 1} \\
\quad+C_{I T M U}^{M 2}+\Delta C_{A S C}^{M 1}+\Delta C_{A S C}^{M 2}= \\
=\frac{L_{M 1} C_{H W}}{\tau_{M 1}}+\frac{L_{M 2} C_{H W}}{\tau_{M 2}}+\frac{l_{M 1} S_{q M U}}{\tau_{M 1}}+\frac{l_{M 2} S_{q M U}}{\tau_{M 2}}+\frac{2 R_{0} S_{A}}{V_{A} \tau_{M 1}}+ \\
+\frac{2 R_{0} S_{A}}{V_{A} \tau_{M 2}}+\frac{l_{M 1} U_{H E}}{\tau_{M 1}}+\frac{l_{M 2} U_{H E}}{\tau_{M 2}}+\Delta C_{A S C}^{M 1}+\Delta C_{A S C}^{M 2}
\end{gathered}
$$

or, after simple transformations

$$
\begin{gathered}
C_{2}=\frac{L_{M 1} C_{H W}+l_{M 1} S_{q M U}+l_{M 1} U_{H E}}{\tau_{M 1}}+\frac{L_{M 2} C_{H W}+l_{M 2} S_{q M U}+l_{M 2} U_{H E}}{\tau_{M 2}}+ \\
+\frac{2 R_{0} S_{A}}{V_{A} \tau_{M 1}}\left(\frac{1}{\tau_{M 1}}+\frac{1}{\tau_{M 2}}\right)+\Delta C_{A S C}^{M 1}+\Delta C_{A S C}^{M 2}
\end{gathered}
$$

at $L_{M 1}>0, L_{M 2}>0, l_{M 1}>0, l_{M 2}>0, C_{H W}>0, S_{q M U}>0$, $\tau_{M 1}>0, \tau_{M 2}>0, R_{0}>0, V_{A}>0, S_{A}>0, U_{H E}>0, \Delta C_{A S C}^{M 1}>0$, $\Delta C_{A S C}^{M 2}>0$.

3. Combined: maintenance-1- at the place of work using a maintenance unit; maintenance- 2 - at the point of maintenance

$$
\begin{gathered}
C_{3}=C_{M U}^{M 1}+C_{M P}^{M 2}+C_{F M U}^{M 1}+C_{F M P}^{M 2}+C_{T M U}^{M 1}+C_{T M P}^{M 2}+ \\
+C_{I T M U}^{M 1}+C_{I T M P}^{M 2}+C_{T D M P}^{M 2}+\Delta C_{A S C}^{M 1}=
\end{gathered}
$$

$$
\begin{gathered}
=\frac{L_{M 1} C_{H W}}{\tau_{M 1}}+\frac{L_{M 2} C_{H W}}{\tau_{M 2}}+\frac{l_{M 1} S_{q M U}}{\tau_{M 1}}+\frac{l_{M 2} S_{q M P}}{\tau_{M 2}}+\frac{2 R_{0} S_{A}}{V_{A} \tau_{M 1}}+ \\
+\frac{2 R_{0} S_{T}}{V_{T} \tau_{M 2}}+\frac{l_{M 1} U_{H E}}{\tau_{M 1}}+\frac{l_{M 2} U_{H E}}{\tau_{M 2}}+\frac{2 R_{0} U_{H E}}{V_{T} \tau_{M 2}}+\Delta C_{A S C}^{M 1}
\end{gathered}
$$

or, after simple transformations

$C_{3}=\frac{L_{M 1} C_{H W}+l_{M 1} S_{q M U}+l_{M 1} U_{H E}}{\tau_{M 1}}+\frac{L_{M 2} C_{H W}+l_{M 2} S_{q M T}+l_{M 2} U_{H E}}{\tau_{M 2}}+$

$$
+2 R_{0}\left(\frac{\tau_{M 1}}{V_{A} \tau_{M 1}}+\frac{S_{T}}{V_{A} \tau_{M 2}}+\frac{U_{H E}}{V_{A} \tau_{M 2}}\right)+\Delta C_{A S C}^{\tau_{M 2}}
$$

at $L_{M 1}>0, L_{M 2}>0, l_{M 1}>0, l_{M 2}>0, C_{H W}>0$, $S_{q M U}>0, S_{q M T}>0, \tau_{T 1}>0, \tau_{M 2}>0, U_{H E}>0, R_{0}>0$, $V_{A}>0, S_{A}>0, V_{T}>0, S_{T}>0, \Delta C_{A S C}^{M 1}>0, \Delta C_{A S C}^{M 2}>0$, where $C_{M P}^{M 1}, C_{M P}^{M 2}, C_{M U}^{M 1}, C_{M U}^{M 2}$ are the specific labor inputs on maintenance-1 and maintenance-2 at their performance at the point of maintenance and with use of the maintenance unit; $C_{F M P}^{M 1}, C_{F M P}^{M 2}, C_{F M U}^{M 1}, C_{F M U}^{M 2}$ - the costs of the same types of service, connected with functioning point of maintenance and the maintenance unit. $C_{T M P}^{M 1}, C_{T M P}^{M 2}, C_{T M U}^{M 1}, C_{T M U}^{M 2}$ are the specific costs of transportation of tractors up to the point of maintenance for performance of the same types of maintenance and also on transportation of the maintenance unit to the place of operation of machines respectively for carrying out maintenance-1 and maintenance-2. $C_{I T M P}^{M 1}, C_{I T M P}^{M 2}$, $C_{I T M U}^{M 1}, \quad C_{I T M U}^{M 2}$ are the specific expenses linked to idle times when carrying out maintenance-1 and maintenance- 2 at point of technical service and with the use of the maintenance unit. $C_{T D M P}^{M 1}, C_{T D M P}^{M 2}$ are the specific expenses linked to the idle times due to transportation of tractors for maintenance-1 and maintenance-2 to the place of service (to the point of maintenance) and back. $\Delta C_{A S C}^{M 1}, \Delta C_{A S C}^{M 1}$ are the additional specific costs of maintenance-1 and maintenance-2, considering increase in labor input and also the expenses connected with losses from idle times of tractors. $L_{M 1}$, $L_{M 1}$ are standard labor input of maintenance-1 and maintenance-2 according to tractor of given brand, manhour. $l_{M 1}, l_{M 2}$ are the standard duration of maintenance1 and maintenance- 2 depending on the tractor's brand, hour. $C_{H W}$ in the hourly compensation to adjuster (operator) taking into account all types of payments, rubles/hour. $S_{q M P}, S_{q M U}$ are specific cost for use of point maintenance and maintenance unit; $\tau_{M 1}, \tau_{M 2}$ is the periodicity of maintenance- 1 and maintenance- 2 , engine hour. $R_{0}$ is the radius of service availability, $\mathrm{km} . V_{T}, V_{A}$ are average transport speed of tractors and maintenance unit, $\mathrm{km} / \mathrm{h} ; S_{T}$ is operating costs on transportation of tractors, rubles/hour. $U_{H E}$ is the hourly expenses (losses from idle times of tractors) linked to idle time on maintenance and with moving of machines to point of maintenance and to the place of work, rubles/hour.

The second stage is a mathematical description of changes in technical maintenance efficiency indicators (labor input and maintenance time). For maintenance-1 they are $\Delta T_{M 1}, \Delta t_{M 1}$; for maintenance-2 they are $\Delta T_{M 2}$, $\Delta t_{M 2}$. The values of these indicators depend on the working conditions of the operator in the field. These elements are included in the mathematical descriptions of $\Delta C_{A S C}^{M 1}$ and $\Delta C_{A S C}^{M 2}$ and are integral elements of mathematical models for the choice of maintenance methods. Then, we write down the change in the labor 
intensity $\left(\Delta T_{M 1}, \Delta T_{M 2},\right)$ and duration $\left(\Delta t_{M 1}, \Delta t_{M 2}\right)$ of the maintenance:

- on labor input -

$$
\begin{aligned}
& \Delta T_{M 1}=T_{M 1}\left[\left(2-U_{f 1}\right) k^{1-U_{f 1}}-1\right], \\
& \Delta T_{M 2}=T_{M 2}\left[\left(2-U_{f 2}\right) k^{1-U_{f 2}}-1\right],
\end{aligned}
$$

- on duration -

$$
\begin{aligned}
& \Delta t_{M 1}=t_{M 1}\left[\left(2-U_{f 1}\right) k^{1-U_{f 1}}-1\right], \\
& \Delta t_{M 2}=t_{M 2}\left[\left(2-U_{f 2}\right) k^{1-U_{f 2}}-1\right],
\end{aligned}
$$

where $\Delta T_{M 1}, \Delta T_{M 2}$ are additional time spent on maintenance-1 and maintenance-2 in the field, manhours; $\Delta t_{M 1}, \Delta t_{M 2}$ are additional tractor downtime for maintenance- 1 and maintenance- 2 in field conditions, hour; $T_{M 1}$ and $T_{M 2}, t_{M 1}$ and $t_{M 2}$ are statutory indicators linking $\Delta T_{M 1}$ and $\Delta T_{M 2}, \Delta t_{M 1}$ and $\Delta t_{M 2}$.

The third stage is a mathematical model for choosing a maintenance method. It is based on expressions (1), (2) and (3). This indicator is used when working simultaneously several brands of tractors - on a formula

$$
C_{C}=\frac{C_{i} \tau_{i}+C_{j} \tau_{j}+C_{k} \tau_{k}+\ldots+C_{m} \tau_{m}}{\tau_{i}+\tau_{j}+\tau_{k}+\ldots+\tau_{m}},
$$

where $C_{C}$ is the weighted specific average cost by maintenance methods, rubles/engine hour; $C_{i}, C_{j}, C_{k}, \ldots C_{m}$ are the specific cost of maintenance i, j, k... for m-brands of tractors, rubles/engine hour; $\tau_{i}, \tau_{j}, \tau_{k}, \ldots \tau_{m}$ are total operating time according to each of these brands of tractors, engine hour.

\section{Results and discussion}

The initial data for calculating the unit cost of tractor maintenance according to the methods refer to agricultural enterprises for which the resource-saving method of maintenance is selected.

They are subdivided into data for this enterprise and for tractor brands. The method provides for obtaining numerical values of the indicator for assessing the choice of methods for tractor maintenance (i-objects). This method allows determining the unit cost of maintenance, either taking into account the additional costs of tractor maintenance with their maintenance in the field conditions, or without taking them into account. The definition of this indicator, taking into account additional costs for maintenance in the field is carried out after the formation of the necessary data.

The procedure for selecting maintenance methods based on working conditions is as follows:

Determine the unit cost of use (rub / man-hours) of the maintenance center for centralized $S_{q M P}^{C e n t}$ and combined $S_{q M P}^{C O m b}$ maintenance, as well as the maintenance unit for decentralized $S_{q M U}^{D e c e n t}$ and combined $S_{q M U}^{C o m b}$ maintenance for the all tractors park - on a formulas (9)-(15):

- centralized maintenance -

$$
\begin{gathered}
S_{q M P}^{C e n t}= \\
=\left[\frac{0.01 \cdot\left(P_{M P}^{B L} K_{M P}^{B L}+P_{M P}^{E Q} E Q\right.}{\left.\sum_{M P}^{E Q}\right)+Z_{M P}^{T E}+Z_{M P}^{E L}+E_{H}\left(K_{M P}^{B L}+K_{M P}^{E Q}\right)}\right] \cdot \gamma
\end{gathered}
$$

$$
S_{q M U}^{\text {Decent }}=
$$

$=\left[\frac{0.01 \cdot\left(P_{M U} K_{M U}+P_{M P}^{E L} K_{M U}^{E L}\right)+Z_{M U}^{T E}+Z_{M U}^{E L}+E_{H}\left(K_{M U}+K_{M U}^{E L}\right)}{\sum_{i=1}^{n} q_{M(1-2)} \tau_{S i} N_{i}}\right] \cdot S_{q M P}^{C e n t}$

- in case of combined maintenance -

$S_{q M P}^{C o m b}=\left[\frac{0.01 \cdot\left(P_{M P}^{B L} K_{M P}^{B L}+P_{M P}^{E Q} K_{M P}^{E Q}\right)+Z_{M P}^{T E}+Z_{M P}^{E L}+E_{H}\left(K_{M P}^{B L}+K_{M P}^{E Q}\right)}{\sum_{i=1}^{n} q_{M(1-2-3)} \tau_{A N i} N_{i}-\sum q_{M(1)} \tau_{S i} N_{i}}\right] \cdot \gamma$

$$
S_{q M U}^{C o m b}=\left[\frac{0.01 \cdot\left(P_{M U} K_{M U}+P_{M P}^{E L} K_{M U}^{E L}\right)+Z_{M U}^{T E}+z_{M U}^{E L}+E_{H}\left(K_{M U}+K_{M U}^{E L}\right)}{\sum_{i=1}^{M} q_{M(1)} \tau_{S i} N_{i}}\right] ;
$$

where $P_{M P}^{B L}, P_{M P}^{E Q}$ are percentages of deductions for depreciation and maintenance of the building and equipment of the technical service center, \%. $K_{M P}^{B L}, K_{M P}^{E Q}$ are capital investments for the construction of the building and for the purchase of equipment for the maintenance station, rub. $Z_{M P}^{T E}, Z_{M P}^{E L}$ are annual labor and cost (for example, electricity and heat energy, water) for the operation of the maintenance station, rub. $P_{M U}, P_{M P}^{E L}$ are percentages of deductions for depreciation and maintenance of the maintenance unit and the building, $\% . K_{M U}, K_{M U}^{E L}$ are capital investments for the purchase of a maintenance unit and for the construction of a building, rub. $Z_{M U}^{T E}, Z_{M U}^{E L}$ are annual labor and equipment costs (electricity, heat and water) for the operation of the maintenance unit and the building, rub. $E_{H}$ is a normative coefficient of capital investment efficiency (according to reference data $-E_{H}=0.15$ ); $q_{M(1)}$ is specific labor intensity of maintenance-1 for one brand of tractor, man-hour/engine hours; $q_{M(1-2)}$ is specific labor intensity of maintenance- 1 and maintenance-2 for one brand of tractor, man-hour/engine hours; $q_{M(1-2-3)}$ is specific labor intensity of maintenance for one brand of tractor, man-hour/engine hours; $\tau_{A N}$ is average annual operating time, engine hours; $\tau_{S i}$ is seasonal operating time, engine hours; $N_{i}$ is a number of tractors, unit.

Where in

$$
\begin{gathered}
q_{M(1)}=\frac{T_{M 1 i}}{\tau_{M 1}}, \\
q_{M(1-2)}=\frac{T_{M 1 i}}{\tau_{M 1}}+\frac{T_{M 2 i}}{\tau_{M 2}}, \\
q_{M(1-2-3)}=\frac{T_{M 1 i}}{\tau_{M 1}}+\frac{T_{M 2 i}}{\tau_{M 2}}+\frac{T_{M 3 i}}{\tau_{M 3}},
\end{gathered}
$$

where $T_{M 1 i}, T_{M 2 i}, T_{M 3 i}$ are regulatory complexity of maintenance-1, maintenance-2, maintenance- 3 for one tractor brand; $\tau_{M 1}, \tau_{M 2}, \tau_{M 3}$ are frequency of maintenance-1, maintenance-2, maintenance-3.

Calculate the radius of maintenance availability by the formula

$$
R_{0}=\delta \sqrt{\frac{F_{s}}{\pi}},
$$

where $\delta$ is the curvature factor of the roads in the vertical and horizontal directions (according to reference data $-\delta=1.5) ; F_{s}$ is serviced area, $\mathrm{km}$.

Determine the hourly operating costs for driving tractors, rub/hours

$$
S_{T}=C_{-} H W+G_{F T} P_{D F},
$$

where $C_{H W}$ is hourly wage, rub/hours; $G_{F T}$ is hourly fuel consumption at driving a tractor, $\mathrm{kg} / \mathrm{h} ; P_{D F}$ - diesel price, rub/liter.

Find the hourly operating costs on the stretch of the maintenance unit, rub/hours

$$
S_{M U}=C_{H M U}+G_{F M U} P_{D M U},
$$


where $C_{H M U}$ is hourly wage of specialist maintenance unit, rub/hours; $G_{F M U}$ is hourly fuel consumption when driving the maintenance unit, $\mathrm{kg} / \mathrm{h} ; P_{D M U}$ is fuel price for maintenance unit, rub/liter.

Calculate losses for tractor idle hour due to maintenance and its move to the maintenance point, rub/hours

$$
D_{S M T U}=D_{T}+D_{A M} \text {, }
$$

where $D_{S M T U}$ is losses for an hour of inactivity of the machine-tractor unit, associated with its maintenance and moving to the maintenance point, rub/hours; $D_{T}$ is losses for an hour idle tractor [7], rub/hours; $D_{A M}$ is losses for an hour idle agricultural machines [7], rub/hours.

Losses for an hour idle tractor [7], rub/hours

$$
D_{T}=\frac{0.01 P_{T} C_{H W}+Z^{T E}+E_{H} C_{H W}}{\tau_{T}},
$$

where $P_{T}$ is the rate of deductions for the renovation of the tractor (can be accepted $=12.5 \%$ ) [8].

Losses for an hour idle agricultural machines [3], rub/hours

$$
D_{A M}=\frac{0.01 P_{A M} C_{H M U}+Z_{A M}+E_{H} C_{H M U}}{\tau_{A M}} .
$$

Weighted average cost of the agricultural machine

$$
C_{H M U}=\frac{C_{H M U i} n_{M U i}+C_{H M U j} n_{M U j}+C_{H M U k} n_{M U k}+\ldots+C_{H M U m} n_{M U m}}{n_{M U i}+n_{M U j}+n_{M U k}+n_{M U m}+\ldots+n_{M U m}}
$$

where $C_{H M U}$ is weighted average cost of the agricultural machine, rub; $C_{H M U i}, C_{H M U j}, C_{H M U k}, \ldots, C_{H M U m}$ are cost of i, j, k... m-brands of agricultural machines, rub; $n_{M U i}, n_{M U j}, n_{M U k}, \ldots, n_{M U m}$ - average number i, j, k... m-brands of agricultural machines.

Perform calculation of additional labor input $\left(\triangle C_{A S C}^{M 1}\right.$, $\Delta C_{A S C}^{M 2}, \Delta C_{A S C}^{M}$ ) on formulas (23), (24), (29), (30).

$$
\begin{aligned}
& \Delta C_{A S C}^{M 1}=\Delta C_{L I}^{M 1}+\Delta C_{I T}^{M 1}, \\
& \Delta C_{A S C}^{M 2}=\Delta C_{L I}^{M 2}+\Delta C_{I T}^{M 2},
\end{aligned}
$$

where $\Delta C_{A S C}^{M 1}, \Delta C_{A S C}^{M 2}$ are increase the unit cost of labor and costs associated with losses from downtime maintenance tractor in field, rub/engine hours.

Additional unit labor costs for maintenance-1 ( $\Delta C_{A S C}^{M 1}$ ) and maintenance-2 $\left(\Delta C_{A S C}^{M 2}\right)$ are formulas:

$$
\begin{aligned}
& \Delta C_{A S C}^{M 1}=\frac{\Delta \mathrm{T}_{\mathrm{M} 1} C_{H W}}{\tau_{\mathrm{M} 1}}, \\
& \Delta C_{A S C}^{M 2}=\frac{\Delta \mathrm{T}_{\mathrm{M} 2} C_{H W}}{\tau_{\mathrm{M} 2}},
\end{aligned}
$$

at $\Delta \mathrm{T}_{\mathrm{M} 1}>0 ; \Delta \mathrm{T}_{\mathrm{M} 2}>0 ; C_{H W}>0 ; \tau_{\mathrm{M} 1}>0 ; \tau_{\mathrm{M} 2}>0$, where $\Delta \mathrm{T}_{\mathrm{M} 1}, \quad \Delta \mathrm{T}_{\mathrm{M} 2}$ are additional expenses on maintenance-1 and maintenance-2 in the field conditions, man hour.

Additional unit costs from losses due to additional downtime for tractor maintenance - at maintenance-1 $\left(\Delta C_{A U C}^{M 1}\right)$ and at maintenance-1 $\left(\Delta C_{A U C}^{M 2}\right)$ - by analogy with (25)-(26) is according to the formulas:

$$
\begin{aligned}
& \Delta C_{A U C}^{M 1}=\frac{\Delta t_{\mathrm{M} 1} D_{S M T U}}{\tau_{\mathrm{M} 1}}, \\
& \Delta C_{A U C}^{M 2}=\frac{\Delta t_{\mathrm{M} 2} D_{S M T U}}{\tau_{\mathrm{M} 2}},
\end{aligned}
$$

at $\Delta t_{\mathrm{M} 1}>0 ; \Delta t_{\mathrm{M} 2}>0 ; D_{S M T U}>0 ; \tau_{\mathrm{M} 1}>0 ; \tau_{\mathrm{M} 2}>0$, where $\Delta t_{\mathrm{M} 1}, \Delta t_{\mathrm{M} 2}$ are additional tractor downtime for maintenance-1 and maintenance-2 in the field conditions, hour; $D_{S M T U}$ is hourly costs of the downtime at maintenance, rub/engine hours.

We will present mathematical descriptions (23) and (24) in expanded form - subject to $\Delta C_{A S C}^{M 1}$ (25), $\Delta C_{A U C}^{M 1}$ (27), $\Delta C_{A S C}^{M 2}(26), \Delta C_{A U C}^{M 2}$ (28):

$$
\Delta C_{A U E L}^{M 1}==\frac{\Delta \mathrm{T}_{\mathrm{M} 1} C_{H W}}{\tau_{\mathrm{M} 1}}+\frac{\Delta t_{\mathrm{M} 1} D_{S M T U}}{\tau_{\mathrm{M} 1}},
$$

at $\Delta \mathrm{T}_{\mathrm{M} 1}>0 ; C_{H W}>0 ; \tau_{\mathrm{M} 1}>0 ; D_{S M T U}>0$;

$$
\Delta C_{A U E L}^{M 2}==\frac{\Delta \mathrm{T}_{\mathrm{M} 2} C_{H W}}{\tau_{\mathrm{M} 2}}+\frac{\Delta t_{\mathrm{M} 2} D_{S M T U}}{\tau_{\mathrm{M} 2}},
$$

at $\Delta \mathrm{T}_{\mathrm{M} 2}>0 ; C_{H W}>0 ; \tau_{\mathrm{M} 2}>0 ; D_{S M T U}>0$.

Additional unit labor costs for maintenance-1 $\Delta C_{A S C}^{M 1}$ and maintenance-2 $\Delta C_{A S C}^{M 2}$ calculated in accordance with the formulas (25), (26).

Additional unit costs from losses due to additional downtime for tractor maintenance - at maintenance-1 $\left(\Delta C_{A U C}^{M 1}\right)$ and at maintenance-1 $\left(\Delta C_{A U C}^{M 2}\right)$ by analogy is by the formulas (27), (28).

Additional expenses on maintenance-1 and maintenance-2 $\left(\Delta \mathrm{T}_{\mathrm{M} 1}, \Delta \mathrm{T}_{\mathrm{M} 2}\right)$ and additional tractor downtime for maintenance-1 and maintenance-2 $\left(\Delta t_{\mathrm{M} 1}, \Delta t_{\mathrm{M} 2}\right)$ in the field conditions - calculated by empirical formulas (found experimentally in agricultural enterprises of the Irkutsk region for the operator's working conditions in the field conditions):

$$
\begin{aligned}
\Delta \mathrm{T}_{\mathrm{M} 1} & =-3.1473 \cdot D_{W C}+3.1491 ; \\
\Delta \mathrm{T}_{\mathrm{M} 2} & =-5.598 \cdot D_{W C}+5.524 \\
\Delta t_{\mathrm{M} 1} & =-1.2611 \cdot D_{W C}+1.263 ; \\
\Delta t_{\mathrm{M} 2} & =-3.511 \cdot D_{W C}+3.357
\end{aligned}
$$

where $D_{W C}$ is the coefficient of working conditions, obtained experimentally in the same enterprises.

The calculation results for the most common brands of tractors are presented in table. 1.

Table 1. Additional unit labor costs for maintenance-1 and maintenance- 2 tractors taking into account the working conditions of the operator in the field conditions

\begin{tabular}{|c|c|c|c|c|c|}
\hline \multirow{2}{*}{ No. } & Brands of tractors & \multicolumn{2}{|c|}{$\begin{array}{c}\text { Additional } \\
\text { labor input: }\end{array}$} & \multicolumn{2}{c|}{$\begin{array}{c}\text { Additional } \\
\text { duration: }\end{array}$} \\
\cline { 3 - 6 } & & $\Delta \mathrm{T}_{\mathrm{M} 1}$ & $\Delta \mathrm{T}_{\mathrm{M} 2}$ & $\Delta t_{\mathrm{M} 1}$ & $\Delta t_{\mathrm{M} 2}$ \\
\hline 1 & $\begin{array}{c}\text { Kirovets, Trion, John Deere, } \\
\text { CLAAS Axion }\end{array}$ & 1.74 & 3.12 & 0.59 & 1.3 \\
\hline 2 & MTZ-80, MTZ-82, MTZ-1221 & 1.26 & 2.17 & 0.51 & 1.25 \\
\hline 3 & T-150K, HZT-150 & 1.67 & 3.31 & 0.16 & 0.56 \\
\hline 4 & DT-75, DT-75M, T-4A & 1.66 & 2.65 & 0.43 & 1.41 \\
\hline
\end{tabular}

Note - values for $\Delta \mathrm{T}_{\mathrm{M} 1}, \Delta \mathrm{T}_{\mathrm{M} 2}$ and $\Delta t_{\mathrm{M} 1}, \Delta t_{\mathrm{M} 2}$ defined for $D_{W C}=0.6$.

7. Determine unit cost $\left(C_{1}, C_{2}, C_{3}\right)$ maintenance- 1 and maintenance-2 with centralized, decentralized and combined maintenance for each brand of tractor according to the formulas (1), (2) and (3).

8 . Based on the unit cost, find the weighted average unit cost of service for all brands of tractors - for the case when in the agricultural enterprise several brands of tractors are used simultaneously - according to the formula (22).

9. Based on the minimum found value of the unit cost of maintenance, a resource-saving method of maintenance is selected from the three calculation results.

The annual economic effect when implementing the selected resource-saving method of maintenance for an individual agricultural enterprise per one average tractor per year is determined by the formulas:

a) in comparison with the method for $C=C_{\max 1^{-}}$

$$
S_{y}=\left(C_{\max 1}-C_{\min }\right) \cdot \tau_{y} \cdot P_{o m},
$$

b) in comparison with the method for $C=C_{\max 2^{-}}$ 


$$
S_{y}=\left(C_{\max 2}-C_{\text {min }}\right) \cdot \tau_{y} \cdot P_{o m},
$$

where $C_{\max 1}, C_{\max 2}$ are unit cost of tractor maintenance by methods, alternative to selected; $C_{\min }$ is unit cost of tractor maintenance according to the selected resourcesaving method; $\tau_{y}$ is average annual operating time of tractors for an agricultural enterprise, hour; $P_{o m}$ is probability of mismatch between the applied and selected maintenance methods.

\section{Conclusion}

Thus, mathematical models for the choice of maintenance methods depending on the unit cost of maintenance indicator (1-3), (4-7) were obtained.

Mathematical models are given for the following maintenance methods: specialized, on the basis of stationary maintenance facilities (centralized) and on the basis of mobile maintenance units (decentralized), as well as a combined one, in which technical maintenance of 1 tractors is carried out at their place of use using maintenance units, and maintenance- 2 - at the central service point where the tractors deliver on their own.

Moreover, mathematical models are presented on the basis of mathematical descriptions of elements whose values depend on the working conditions of the operator. In conclusion, a generalized mathematical model (8) has been developed for each maintenance method as a weighted average unit cost of maintenance. The values of the additional labor costs for maintenance (labor input and duration of maintenance-1 and maintenance-2) of tractors were obtained experimentally, taking into account the working conditions of the operator in the field, which was done according to the model of the MTZ tractor (table 1).

For other tractor models - on the basis of the similarity method under the assumption that the additional labor input and duration of maintenance, as well as the coefficient of the operator's working conditions in the field, are identical for all tractor models. It was found that the labor costs for maintenance- 1 and maintenance- 2 of tractors depend on the working conditions of the operator in the field and are described by empirical formulas that have the form of linear decreasing functions.
The annual economic effect of applying this methodology, calculated through the example of agricultural enterprises in the Irkutsk region, is 27.8 thousand rubles per tractor. The method was tested at farms in the Tulunsky and Cheremkhovsky districts of the Irkutsk region and can be useful to engineers maintaining tractors at farms. The results of the study can be used in agricultural enterprises, both the Irkutsk region and the Siberian region as a whole.

\section{References}

1. V.N. Khabardin, Resource-saving technologies, methods and means of tractor maintenance, Monograph (Irkutsk State Agricult. Acad., Irkutsk, 2009), 384 p.

2. R. Khodabakhshian, A review of maintenance management of tractors and agricultural machinery: Preventive maintenance systems, Agricult. Eng. Int.: CIGR J. 15, 147-159 (2013)

3. A. Rohani, Prediction of tractor repair and maintenance costs using Artificial Neural Network, Expert Syst. with Applicat. 38, 8999-9007 (2011)

4. C.J.A. Ter Berg, Expert judgement based maintenance decision support method for structures with a long service-life, Struct. and infrastruct. Engineer. 15(4), 492-503 (2019)

5. E.I. Kubeev, Features of the direction of reducing the complexity of maintenance of automotive machinery, in: Proc. of the Int. Acad. of Agricult. Ed. 38, 9-13 (2018)

6. N.V. Chubareva, Resource-saving by choosing methods of tractors maintenance, in: Conf. Ser. Mater. Sci. and Engineer. 632(1), 012045 (2019)

7. I.P. Tersky, Calculation and selection of organizational forms and means of technical maintenance of the machine and tractor fleet in the department (team) of an agricultural enterprise using a personal computer (Irkutsk, 2002), p. 37

8. D.M. Voronin, Forecasting operating costs for equipment maintenance, Mechanizat. and electrificat. 4, 60-66 (2009) 